\title{
A Summary of recent Updates in the Search for Cosmic Ray Sources using the IceCube Detector
}

\author{
The IceCube Collaboration ${ }^{\dagger}$ \\ E-mail: tessa.carvereunige.ch
}

In 2012 the IceCube detector observed the first clear detection of a diffuse astrophysical high energy neutrino flux, however, the sources for these neutrinos have yet to be found. Hadronic interactions around cosmic ray accelerators result in both high energy gamma and neutrino fluxes from the decay of neutral and charged pion respectively. Observing gamma ray sources with neutrinos provides unique evidence of the hadronic nature of these sources. These searches involve looking for a significant clustering of neutrinos from any direction or a correlation with a known source observed by other messengers also expected to emit a neutrino flux. These searches range from stacking populations of similar sources such as active galactic nuclei, to time-dependent searches targeting individual sources when they are in flaring states. We present here the most recent updates in the search for sources of extraterrestrial neutrinos using the latest methods and source information.

Corresponding author: T. Carver*1

${ }^{1}$ Ecole du Physique, Universite de Geneve, Switzerland

The European Physical Society Conference on High Energy Physics

5-12 July, 2017

Venice

${ }^{*}$ Speaker. 


\section{Introduction}

For hundreds of years astrophysicists learnt about what lies outside of the atmosphere using optical observations of objects emmiting particles with energies of approximately few eV. Advancements in technology and astrophysics have allowed observations to span a much larger range of the electro-magnetic spectrum up to $100 \mathrm{TeV}$ with the advent of the multi-wavelength astronomy, and to expand the field with multi-messenger observations including Cosmic Rays (CR), Gravitational Waves (GW) and neutrinos.

The advantage of using neutrinos to determine CR sources is that their horizon is of the scale of the Universe, unlike high energy photons that have a limited horizon. In addition neutrinos are also neutral particles which are not deflected by magnetic fields.

The IceCube detector consists of instrumentalized antarctic ice [11,10]. Within the ice there are 86 strings, each with 60 digital optical modules that measure Cherenkov radiation induced by charged particles resulting from the neutrino deep inelastic scattering with nuclei surrounding the detector. By registering the energy deposited from this light and the timing, a track can be reconstructed to give an estimate of the original neutrino direction and energy using methods described in $[14,17]$. At the high energies on the TeV scale probed by IceCube, the direction of the charged lepton track corresponds very closely to the direction of the original neutrino path.

IceCube events form two main topologies. The first being track-like events which are the observations of high energy muons propagating through the ice. These muons originate from cosmic ray interactions in the atmosphere which is a large source of background, or charged current $v_{\mu}$ interactions. These events can have an angular resolution of less than $1^{\circ}$ but a poor energy resolution as the event is not contained within the detector volume and so it is impossible to be sure of the total energy deposited in the ice. Alternatively, neutral current $v_{\mu}$ interactions or charged current interactions from other flavours of neutrinos, produce much more spherical topologies known as cascade events. The angular resolution of these events is poor, on the order of $10^{\circ}$, as the only real indication of direction comes from the timing. However, cascade events can have a high energy resolution as they are largely contained in the detector.

\section{Diffuse Neutrino Flux}

The first direct evidence of an astrophysical neutrino flux was observed by IceCube $[4,2]$ with a selection of events designed to optimize the fraction of astrophysical events. The flux of this sample compared with the best fit of simulated background atmospheric muons and atmospheric signal can be seen in Fig 1. This selection left a total of 52 events over 4 years where only $20 \pm 6$ of those would be expected from an atmospheric flux alone therefore rejecting an atmospheric only model at more than $5 \sigma$. An unbroken power law fit applied to this sample gives a spectral index of $-2.58 \pm 0.25$.

A larger sample of high quality track events are selected $[6,7,1]$ which is several orders of magnitidue larger with thousands of events per year. However, a selection starting at $1 \mathrm{TeV}$ becomes dominated by muon tracks from atmospheric neutrinos. A fit of the conventional atmospheric, prompt, and astrophysical flux for this selection of through going tracks using 8 years of 


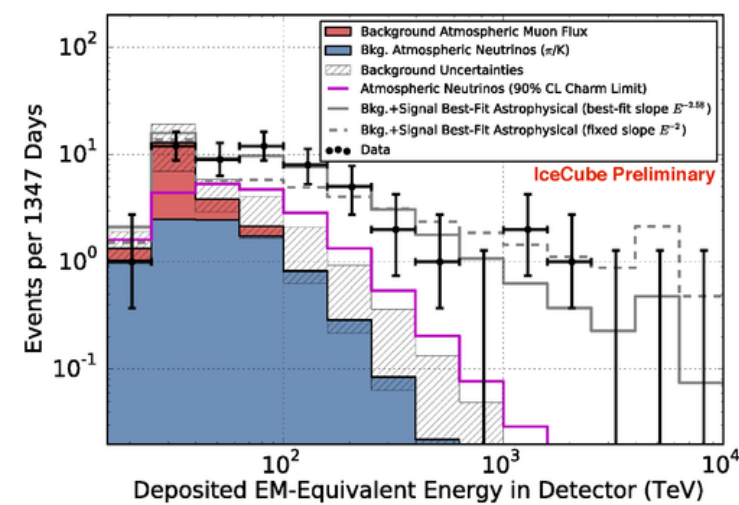

Figure 1: Simulated background and astrophysical neutrino fluxes compared with that observed using 4 years of IceCube data with the High Energy Starting Event selection. This was the first direct evidence for an astrophysical neutrino flux.

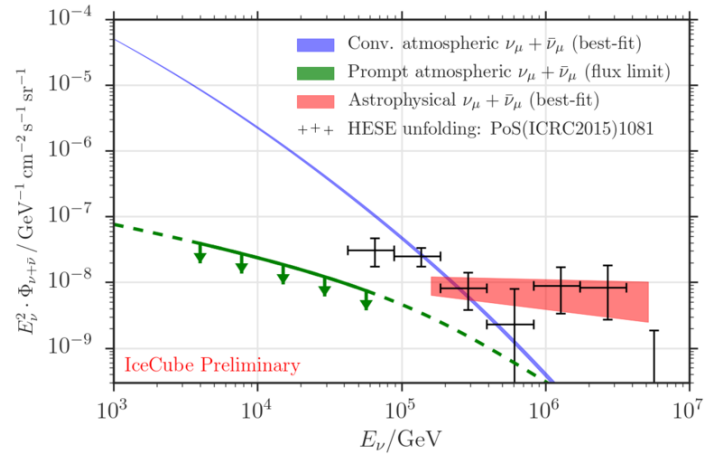

Figure 2: The fit results of different components of a diffuse neutrino flux compared with 8 years of IceCube data for a selection of principallly throughgoing tracks.

IceCube data [1] can be seen in Fig 2. The resulting astrophysical fit was found to be :

$$
\Phi_{v+\bar{v}}=1.01_{-0.23}^{+0.26} \times\left[\frac{E_{V}}{100 \mathrm{TeV}}\right]^{-2.19 \pm 0.10}
$$

There is a slight tension between the softer fit in the Combined diffuse flux and the lower energy throughgoing track sample. This tension could be explained by a possible break in the flux of a prompt component which would result different unbroken spectral fits for different energy windows.

\section{Determining Neutrino Flux Sources}

\subsection{Unbinned Maximimum Likelihood Method}

To establish whether or not a certain selection of events is correlated significantly with certain source positions compared with the isotropic background, an unbinned maximum likelihood method as described in [15] is used:

$\mathscr{L}\left(n_{s}, \gamma\right)=\prod_{j} \prod_{i \in j}\left[\frac{n_{s} \cdot w_{j}(\gamma)}{N_{j}} S^{\mathrm{spat}}\left(\vec{x}_{i}, \vec{x}_{s}, \sigma_{i}\right) S_{j}^{\mathrm{ener}}\left(E_{i}, \gamma\right)+\left(1-\frac{n_{s} \cdot w_{j}(\gamma)}{N_{j}}\right) B_{j}^{\mathrm{spat}}\left(\vec{x}_{i}\right) B_{j}^{\mathrm{ener}}\left(E_{i}\right)\right] \cdot P\left(\gamma \mid \gamma_{\text {astro }}\right)$

In this equation $j$ represents each subsample and $i$ an event within this sample. The properties of these events, $E_{i}, \bar{x}_{i}, \sigma_{i}$ represent the reconstructed event energy, direction and estimated angular uncertainty respectively. The probability distribution functions of the background and signal are then $B$ and $S$. These functions are separated into spatial an energy components. The spatial background probability $B_{j}^{\text {spat }}$ is uniform in right ascension. Whereas the probability $S_{j}^{\text {spat }}$ is defined by convolving the detector acceptance with a neutrino emmission model or hypothesis. The background probability can either be estimated assuming a background dominated event selection and scrambling the events in right ascension, or by using simulated atmospheric muon and neutrino events. The signal probability is neccessarily calculated by using simulated neutrino induced 


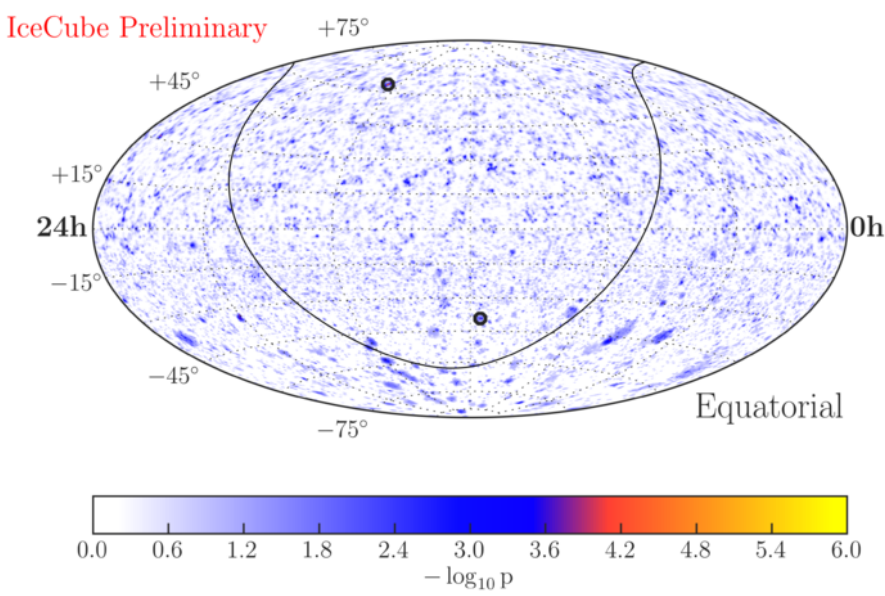

Figure 3: 7 year + MESE SkyMap in equatorial coordinates. The plot shows pre-trial p-Values for all spots in the sky except for a declination region of 5 degrees around the poles.

muon tracks weighted to an astrophysical spectrum. The number of signal events $n_{s}$ from this source model and the spectral index from this signal $\gamma$, are set as free parameters to be fit when the likelihood is maximised.

\subsection{Time Integrated Point Source Search}

Assuming that a significant fraction of the astrophysical neutrino flux originates from prominant point sources we can search for a potential neutrino source by looking for a significant clustering in the direction of well constructed track events. This method not only assumes no prior knowledge on the position of these sources but also can be applied to a large sample dominated by atmospheric neutrino background. This is because the atmospheric background should be uniform in direction where as the signal would cluster around source positions. The use of a large sample begining at $1 \mathrm{TeV}$ is beneficial as it includes a much higher rate of astrophysical events giving a higher chance for events to cluster. The hottest spots for a sample of high quality tracks starting from about $1 \mathrm{TeV}$ over 7 years of IceCube data in the northern and southern sky respectively are highlighted in Fig 3. The post trial p values for each of these hotspots yielded only $44 \%$ and $39 \%$ respectively [9] which are compatible with the background hypthesis.

\subsection{Galactic Plane Search}

In the case where the astrophysical neutrino flux is not dominated by individual point sources, another possibilty for the spatial signal distribution could be that most of the flux originates from the Galactic plane. Multiple models of neutrino emission from the plane can be tested as a signal map. One such model based on the KRA-gamma model with a $50 \mathrm{PeV}$ CR cutoff [16] can be seen in Fig 4. The IceCube upper limit at a 90\% confidence level for this model is shown in Fig 5 . This limit can be directly compared with the measured combined diffuse astrophysical flux [5] and the measurements from northern sky throughgoing muon tracks [7]. IceCube is able to set strong limits 
of the contribution of the plane and has shown that a maximum of $14 \%$ of the observed isotropic diffuse emmision come from the Galactic plane for the case of a Fermi-LAT [12] template assuming gamma-rays from pion decay with an $E^{-2.5}$ power law.

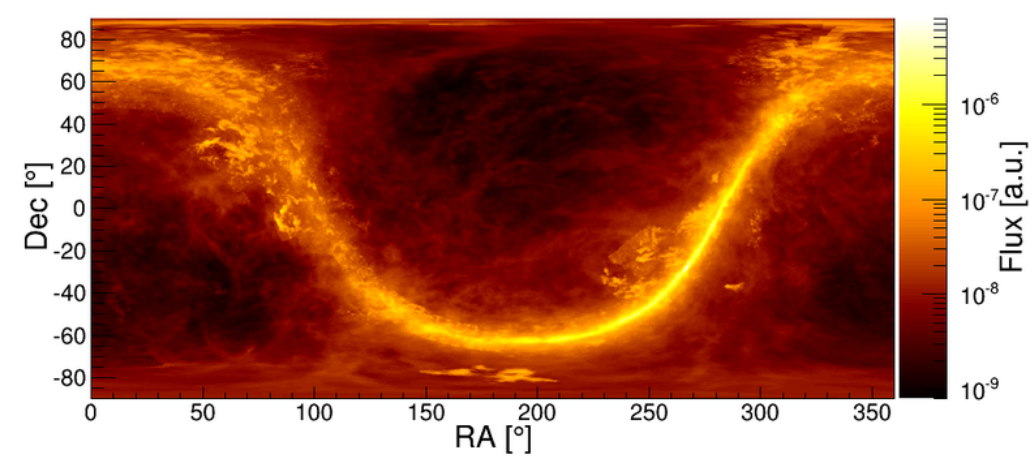

Figure 4: Model Spatial Template: KRA-gamma with $50 \mathrm{PeV} \mathrm{CR} \mathrm{cutoff.} \mathrm{Equatorial} \mathrm{coordinates.}$

\subsection{Time Dependent Source Search}

Neutrino sources could also vary in time in the form of flares. A significant clustering of IceCube events in space and also time would indicate the presence of a flaring source. This can be implemented by adding a time component to the signal and background probability functions. In this case the background probability is uniform in time while the signal temporal component is modeled as a Gaussian.

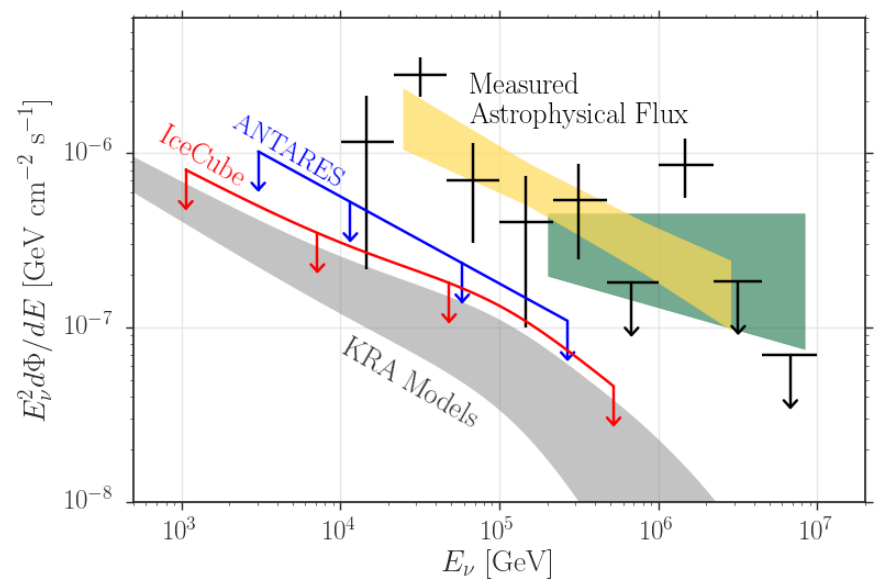

Figure 5: Upper limits on the three-flavor neutrino flux from the Galaxy with respect to KRA model predictions and the measured astrophysical flux. The energy range of validity is from $1 \mathrm{TeV}$ to $500 \mathrm{TeV}$, calculated by finding the energy range that gives $90 \%$ of the sensitivity. The ANTARES limit in blue [13], has been scaled to represent an all-sky integrated flux for comparison. For comparison, measurements of the all-sky diffuse flux are shown: a differential unfolding (black points) and a power-law unfolding (yellow band) of combined IceCube data sets [5] as well as a measurement based only on northern sky muon data (green band) [7]. 


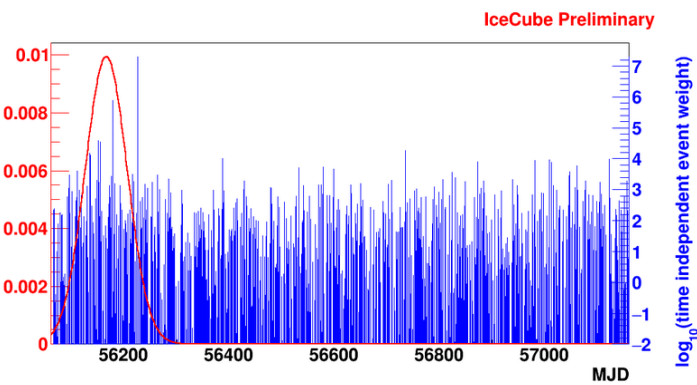

Figure 6: Time Dep northern Sky gaussian best fit

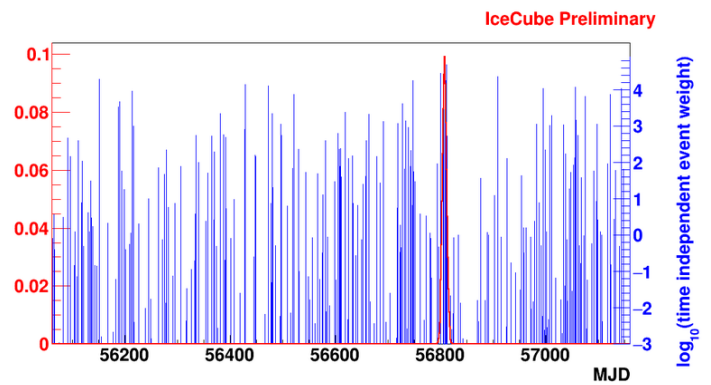

Figure 7: Time Dep northern Sky gaussian best fit

For an untriggered analysis an allsky scan is conducted with no priors on the timing of the neutrino events. A clustering in time and space is compared with equivalent tests using many trials of scrambled background, just as is done for the time integrated case. The Gaussain best fit compared with the weighted IceCube events as a function of time is seen in Fig 6 and Fig 7 for both the northern and the southern skies. The post-trial p-values are $12.6 \%$ and $22.4 \%$ respectively, both compatible with background fluctuations.

\subsection{Ultra High Energy Cosmic Ray Correlation}

Another method in the search for the origins of the astrophysical neutrino flux that differs from searching for a clustering of events or a direct correlation with a known source, is to search for a correlation of IceCube neutrino events with events from another messenger particle thought to be produced by the same source. One such analysis conducted at IceCube was a correlation between Ultra High Energy Cosmic Ray (UHECR) events above $50 \mathrm{EeV}$ observed by Telescope Array (TA), the Pierre Auger Observatory and the high energy neutrino events from IceCube. The unbinned maximum likelihood method was implemented using a relatively pure sample of neutrino events as the signal probability map and assuming deflection angles of $3^{\circ}, 6^{\circ}$, and $9^{\circ}$. The most significant result from this search was found using IceCube cascade events and assuming $6^{\circ}$ cosmic ray deflection. This result has fallen from $3 \sigma$ [8] to $2 \sigma$ [3] with the addition of 2 more years of high energy starting events [2]. These results are in agreement with an independant correlation analysis of the same events without assuming any prior deflection model.

\section{Conclusion}

IceCube has observed a diffuse astrophysical flux and there are continued efforts to determine more precise spectral information regarding this flux. However, the sources for this flux have yet to be resolved. Many methods have been applied which involve searching for a clustering in space and time or a correlation with sources and events observed by other telescopes.

In an effort to combine information from multiple particle messangers to maximise our source information IceCube has implemented real time alerts which send track information for particualrly high energy events. The aim is to trigger other telescopes and observe more information on sources flaring in real time. 
There are also plans to upgrade IceCube expanding the effective area in particular for high energy events. With a higher event rate the sources of the astrophysical signal should become possible to resolve providing unique information on the sources of cosmic rays.

\section{References}

[1] A measurement of the diffuse astrophysical muon neutrino flux using eight years of IceCube data. PoS, ICRC2017:1005.

[2] Observation of Astrophysical Neutrinos in Six Years of IceCube Data (HESE-6year). PoS, ICRC2017:981.

[3] Search for correlations in the arrival directions of astrophysical neutrino candidates and ultra-high energy cosmic rays. PoS, ICRC2017:961.

[4] M. G. Aartsen et al. Evidence for high-energy extraterrestrial neutrinos at the icecube detector. Science, 342(6161), 2013.

[5] M. G. Aartsen et al. A combined maximum-likelihood analysis of the high-energy astrophysical neutrino flux measured with IceCube. Astrophys. J., 809(1):98, 2015.

[6] M. G. Aartsen et al. Evidence for Astrophysical Muon Neutrinos from the Northern Sky with IceCube. Phys. Rev. Lett., 115(8):081102, 2015.

[7] M. G. Aartsen et al. Observation and characterization of a cosmic muon neutrino flux from the northern hemisphere using six years of icecube data. Astrophys. J., 833(1):3, 2016.

[8] M. G. Aartsen et al. Search for correlations between the arrival directions of IceCube neutrino events and ultrahigh-energy cosmic rays detected by the Pierre Auger Observatory and the Telescope Array. JCAP, 1601(01):037, 2016.

[9] M. G. Aartsen et al. All-sky search for time-integrated neutrino emission from astrophysical sources with 7 yr of icecube data. Astrophys. J., 835(2):151, 2017.

[10] M.G. Aartsen et al. The icecube neutrino observatory: instrumentation and online systems. JINST, 12(03):P03012, 2017.

[11] A. Achterberg et al. First year performance of the icecube neutrino telescope. Astropart. Phys., 26(3): $155-173,2006$.

[12] M. Ackerman et al. Fermi-LAT Observations of the Diffuse $\gamma$-Ray Emission: Implications for Cosmic Rays and the Interstellar Medium. ApJL, 750:3, 2012.

[13] S. Adrián-Martínez et al. Constraints on the neutrino emission from the Galactic Ridge with the ANTARES telescope. Physics Letters B, 760:143-148, 2016.

[14] J. Ahrens et al. Muon track reconstruction and data selection techniques in amanda. Nucl. Instrum. Meth. A, 524(1):169 - 194, 2004.

[15] Jim Braun et al. Methods for point source analysis in high energy neutrino telescopes. Astropart. Phys., 29(4):299 - 305, 2008.

[16] D. Gaggero et al. The Gamma-Ray and Neutrino Sky: A Consistent Picture of Fermi-LAT, Milagro, and IceCube Results. ApJL, 815:L25, 2015.

[17] K. Schatto. Stacked searches for high-energy neutrinos from blazars with IceCube. PhD thesis, Johannes Gutenberg-Universität, 2014. 